\title{
Review Article \\ Deep Brain Stimulation in Parkinson's Disease: New and Emerging Targets for Refractory Motor and Nonmotor Symptoms
}

\author{
Dustin Anderson, Grayson Beecher, and Fang Ba \\ Department of Medicine, University of Alberta, Edmonton, AB, Canada T6G 2G3 \\ Correspondence should be addressed to Fang Ba; fb@ualberta.ca
}

Received 5 February 2017; Revised 12 May 2017; Accepted 6 June 2017; Published 6 July 2017

Academic Editor: Aparna Wagle Shukla

Copyright (C) 2017 Dustin Anderson et al. This is an open access article distributed under the Creative Commons Attribution License, which permits unrestricted use, distribution, and reproduction in any medium, provided the original work is properly cited.

\begin{abstract}
Parkinson's disease (PD) is a progressive neurodegenerative condition characterized by bradykinesia, tremor, rigidity, and postural instability (PI), in addition to numerous nonmotor manifestations. Many pharmacological therapies now exist to successfully treat PD motor symptoms; however, as the disease progresses, it often becomes challenging to treat with medications alone. Deep brain stimulation (DBS) has become a crucial player in PD treatment, particularly for patients who have disabling motor complications from medical treatment. Well-established DBS targets include the subthalamic nucleus (STN), the globus pallidus pars interna (GPi), and to a lesser degree the ventral intermediate nucleus (VIM) of the thalamus. Studies of alternative DBS targets for PD are ongoing, the majority of which have shown some clinical benefit; however, more carefully designed and controlled studies are needed. In the present review, we discuss the role of these new and emerging DBS targets in treating refractory axial motor symptoms and other motor and nonmotor symptoms (NMS).
\end{abstract}

\section{Introduction}

Parkinson's disease (PD) is a common neurodegenerative condition. Many successful pharmacological therapies and strategies have been developed to treat both the motor and nonmotor manifestations of PD; however, as PD progresses it often becomes intractably difficult to treat, typically as a result of motor complications related to treatment. Since the seminal study by Benabid et al. targeting the ventral intermediate nucleus (VIM) of the thalamus [1], deep brain stimulation (DBS) has emerged as a key player in the treatment of PD. Multiple randomized controlled studies have demonstrated subthalamic nucleus- (STN-) and globus pallidus interna- (GPi-) DBS to be superior to medical treatment alone in treating a number of the cardinal symptoms and motor complications from therapy [1-3]. The benefit of DBS on axial symptoms is less clear. Several reports have indicated improvement of posture, gait, and balance control after STN- or GPi-DBS, when these symptoms were responsive to levodopa treatment before DBS surgery [4-9]; however, the benefit on postural instability (PI) and gait is not sustained [4]. Moreover, it has been noted that a significant number of patients report postoperative worsening of gait, despite concurrent improvement in motor scores and global outcomes after bilateral STN-DBS. Further, fall risk has been demonstrated to increase and levodopa-resistant freezing of gait (FoG) persists or worsens [10-16]. The axial domains of speech [17-19] and swallowing [20,21] have also shown to be impacted by DBS. To complicate matters further, stimulation parameters (i.e., high frequency stimulation) can also lead to adverse axial effects in patients. These disparities in outcome have fueled the exploration for novel DBS targets that may prove beneficial at treating the axial motor symptoms of PD. In addition to refractory axial motor symptoms, it is clear that nonmotor symptoms (NMS) can also become particularly troublesome [22], as PD progresses and increases in severity. NMS have a significant impact on prognosis and quality of life [23], again highlighting the need for alternative DBS targets that will have therapeutic benefit not only for refractory motor symptoms, but for NMS in PD as well. 
In the present review, we discuss new and emerging DBS targets currently being investigated for the treatment of refractory motor symptoms and NMS in PD. These targets include the pedunculopontine nucleus (PPN), the caudal zona incerta (ZI), the substantia nigra $(\mathrm{SN})$ pars reticulate $(\mathrm{SNr})$ (Figure 1), the motor cortex, and other less explored targets.

\section{New and Emerging DBS Targets for Refractory Motor Symptoms}

2.1. Refractory Tremor. For tremor-dominant PD, where severe and disabling tremor is refractory to treatment, VIMDBS has been shown to suppress tremor effectively. In addition, STN- and GPi-DBS both provide sustained benefit for $\mathrm{PD}$ resting tremor. For severe tremor and coexisting essential tremor, DBS leads implanted in the posterior aspect of the GPi or STN (i.e., ZI region bordering the STN) appear to be of benefit [23].

2.1.1. Caudal Zona Incerta. The ZI is a small heterogeneous cellular nucleus that lies within the anatomical location termed the posterior subthalamic area (PSA) [24, 25]. The borders of the PSA include the posterior border of the STN anteriorly, the dorsal SN inferiorly, the ventral thalamic nuclei superiorly, the anterolateral red nucleus posteromedially, the medial lemniscus posteriorly, and the internal capsule laterally $[24,25]$. The rostral ZI lies along the dorsal and medial STN, while the caudal ZI (cZI) is located posteromedially to the STN [26] (Figure 1(b)). Various functions of the ZI have been postulated throughout the literature; however, it is commonly held that the ZI plays a role in visceral function, arousal, attention, and posture and locomotion, with the cZI mediating the latter [26]. The cZI has widespread afferent and efferent projections amongst the cerebral cortex, diencephalon, brainstem, cerebellum, and spinal cord, the majority of which are GABAergic [26]. While its circuitry remains complex and poorly understood, it is postulated that the cZI may act as an integrator within and between the basal ganglia-thalamocortical loop and the cerebellothalamocortical loop, assisting in the synchronization of oscillatory neuronal firing in both of these pathways [27]. Abnormalities in oscillatory neuronal synchronization that are generated along either of these loops or at the level of the cZI are thought to play a major role in the generation of tremor $[24,25,27]$.

The benefit of cZI-DBS for tremor control has been well established in studies investigating its role in essential tremor [28]. In PD, the majority of information that has been gleaned regarding the cZI has come from lesional studies. It has previously been shown that subthalamotomy including the region of the $\mathrm{ZI}$ can lead to clinical improvement in PD [29]. Subsequent work focusing on the ZI and the cZI has led to significant discoveries regarding the promise of this structure as a DBS target in PD [24, 25]. The relevance of the cZI as a DBS target in PD was brought to the forefront by Plaha et al., in their study comparing motor outcomes amongst three DBS targets: the CZI, the posterodorsal STN, and dorsomedial/medial STN [30]. When compared to STN stimulation, unilateral cZI stimulation with mean frequency of $150 \mathrm{~Hz}$ led to greater improvement in tremor control and overall Unified Parkinson's Disease Rating Scale (UPDRS) motor scores.

A subsequent longitudinal, observational study by Plaha et al. again demonstrated the utility of cZI-DBS (bilateral, $145 \mathrm{~Hz}$ ) in reducing parkinsonian tremor, as well as a variety of other tremor types, including cerebellar outflow, essential, and dystonic tremor at 12 months of follow-up [27]. Recent work by Blomstedt et al., in an open labeled study with 18 months of follow-up [23-25], echoed the results of Plaha et al. [27], demonstrating the benefit of unilateral cZI-DBS with mean frequency of $160 \mathrm{~Hz}$ in the treatment of contralateral, severe parkinsonian tremor. The benefit on rigidity and bradykinesia was not as profound as in STN-DBS; however, a number of studies have suggested that cZI-DBS has a lower incidence of speech deterioration and is associated with better neuropsychological outcomes [27, 31]. That being said, cZI-DBS is not as well established as STN- or GPi-DBS in PD. Further larger scale studies are required to guide future target selection.

2.1.2. Centromedian and Parafascicular Nuclei. The centromedian and parafascicular nuclei (CMPf) (Figure 1(c)) are the two main constituents of the intralaminar nucleus of the thalamus and have several connections within the basal ganglia, with projections to the STN, substantia nigra (SN), and GPi [32]. It has been postulated that CMPf-DBS affects other thalamic components [ventralis oralis anterior (VOA) and VIM] whose role in tremor control has been well established $[33,34]$.

Interest in the CMPf as a DBS target resurfaced following the observation by Krauss et al. that stimulation of CMPf appeared to abolish resting tremor in 1 patient and involuntary choreoathetotic and dyskinetic movements in 2 others [35]. In subsequent reports, it was observed that CMPf stimulation, independent of STN stimulation, led to reduction of tremor-related muscle activity in 2 patients with PD [36, 37]. Additionally, they demonstrated better tremor control compared with STN-DBS alone. Mazzone et al. [38] demonstrated that combination of CMPf- and GPiDBS reduced UPDRS III scores by $49.9 \%$, a value significantly different when compared to CMPf or GPi stimulation alone. Unfortunately, tremor control was not specified within the study. Further studies should help clarify whether CMPf stimulation is superior to VIM-DBS for tremor control in PD.

\subsection{Refractory Axial Motor Symptoms-Gait and Balance.} FoG, in addition to other gait disturbances such as decreased stride length and gait variability, is associated with increased fall risk in patients with $\mathrm{PD}$ [50]. These symptoms are typically refractory to therapy, including STN- and GPi-DBS $[51,52]$, and are thus a significant source of morbidity in PD [53]. The pathophysiology and neuropathological substrates underlying FoG remain largely unknown. FoG may be due to a failure to adequately scale amplitudes for the intended movement [54] and/or defective motor programming by the supplementary motor area (SMA) and its maintenance by the basal ganglia, leading to a mismatch between intention and automation [54]. 


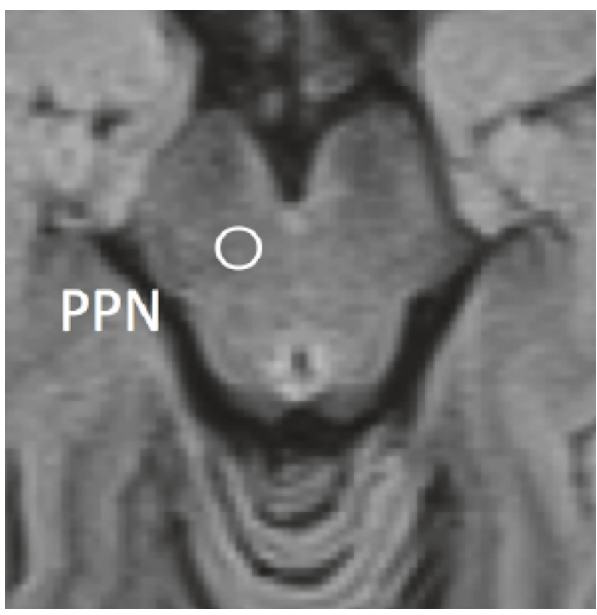

(a)

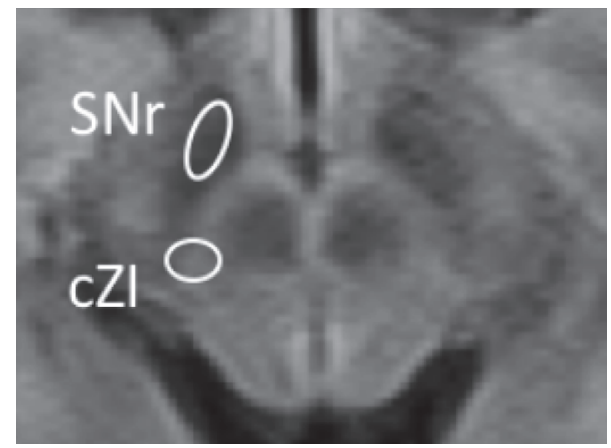

(b)

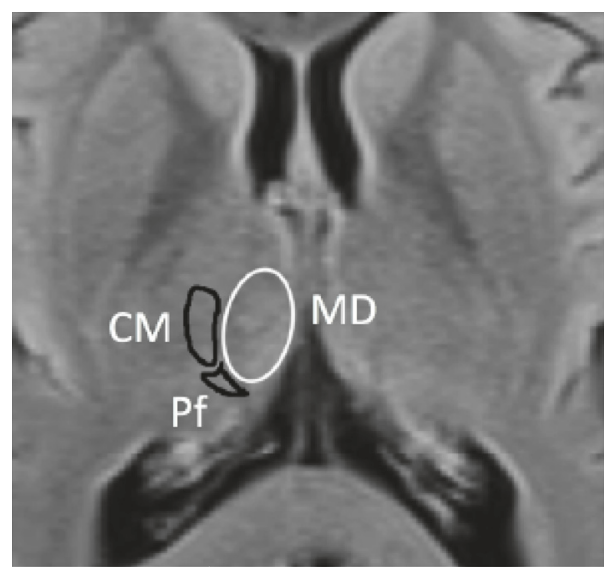

(c)

FIGURE 1: Axial MRI imaging at the level of the midbrain and thalamus, demonstrating the anatomical locations of DBS targets described in the review. CMPf, centromedian-parafascicular nuclear complex; cZI, caudal zona incerta; PPN, pedunculopontine nucleus; SNr, substantia nigra pars reticulata.

2.2.1. Pedunculopontine Nucleus. The mesencephalic locomotor region (MLR) appears critical for normal gait function [61]. The PPN is a key component of the MLR [62] (Figure 1(a)). Widespread projections involving the PPN include direct glutamatergic inputs from the motor cortex and GABAergic inputs from SNr, GPi, STN, and deep nuclei of the cerebellum. Ascending efferent projections target GPi, SN pars compacta [63], and thalamus. Descending efferent projections target pontine and medullary reticular formations, as well as spinal cord structures vital to the control of muscle tone and locomotion. The PPN appears to play a key role in the initiation, acceleration, deceleration, and termination of locomotion through connections to the basal ganglia and higher cortical regions [61]. PPN neuronal loss is evident in PD [64]. Ways to modulate PPN connectivity and activity have proven elusive. Acetylcholinesterase inhibitors may affect the PPN but effects are likely modest.

Jenkinson et al. were the first group to demonstrate the efficacy of PPN-DBS, in a 1-methyl-4-phenyl-1,2,3,6tetrahydropyridine- (MPTP-) exposed macaque [65]. Following MPTP exposure, unilateral PPN stimulation was equivalent to levodopa in improving motor activity scores [65]. In 2005, 2 case studies were the first to establish the safety and efficacy of PPN-DBS in humans [39, 40] (Table 1), demonstrating improvements in UPDRS motor scores. Subsequently, a study by Plaha and Gill was the first to show the role of PPN-DBS in improving gait dysfunction and PI in PD [40]. Multiple open labeled PPN-DBS studies have demonstrated clinical improvement in patients with $\mathrm{PD}$, although results have been variable [41, 42, 45, 47] (Table 1). Additional open labeled studies from Thevathasan et al. [45, 46, 66, 67] demonstrated that PPN stimulation $(20-35 \mathrm{~Hz})$ improved frequency of falls in PD patients with severe FoG and PI during the "on" state [45]. One study showed improvement in gait and falls questionnaire score but not UPDRS III score in 5 patients with PD implanted with bilateral PPN electrodes [46]. The first double-blinded assessment of PPN-DBS was performed by Ferraye and colleagues [43], demonstrating improvement in FoG but not PI or overall UPDRS scores. The lack of improvement in global motor function and axial symptoms, other than FoG, was in opposition to previous studies (Table 1) [39-41, 46, 48]. Moro 


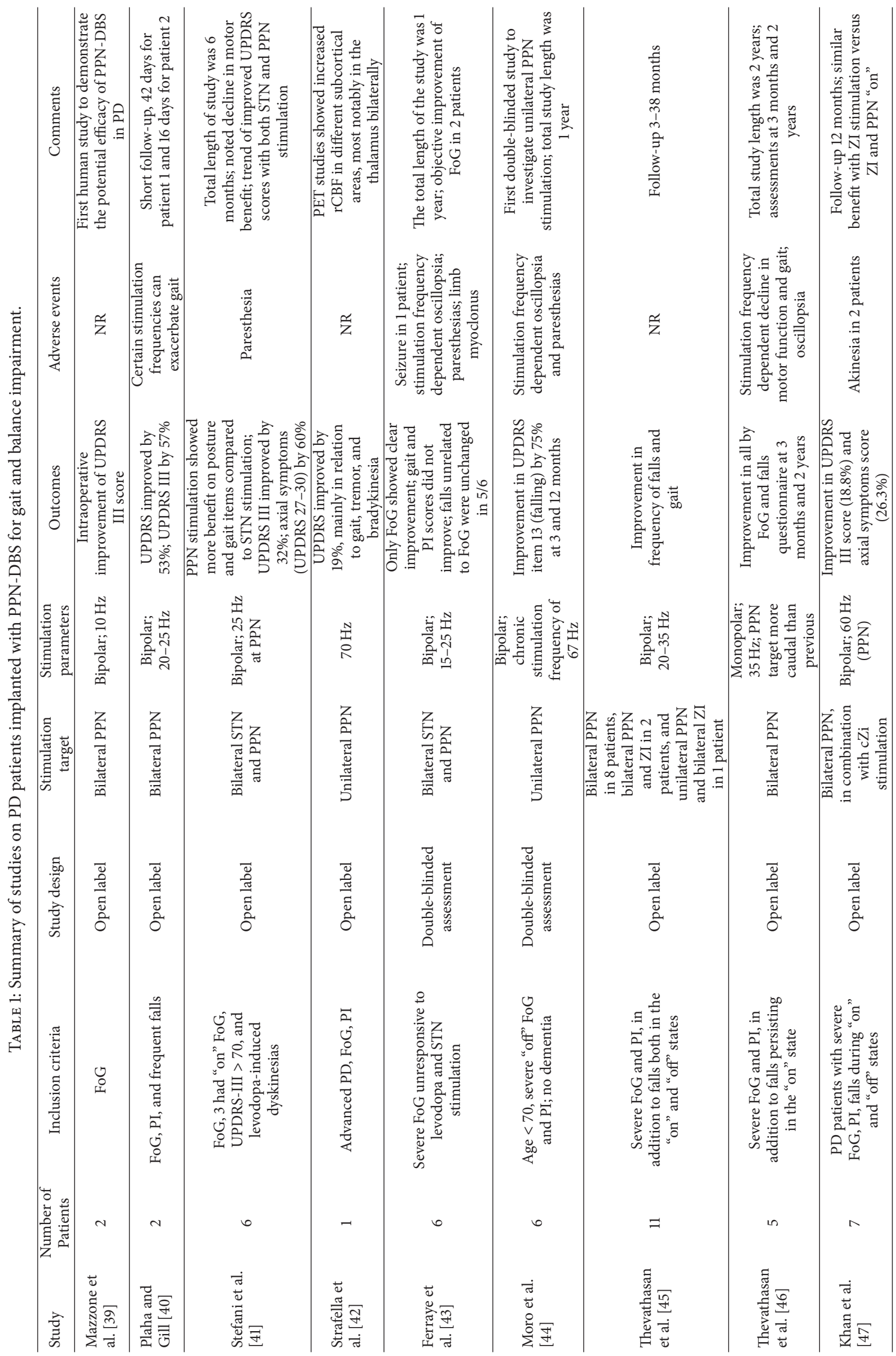




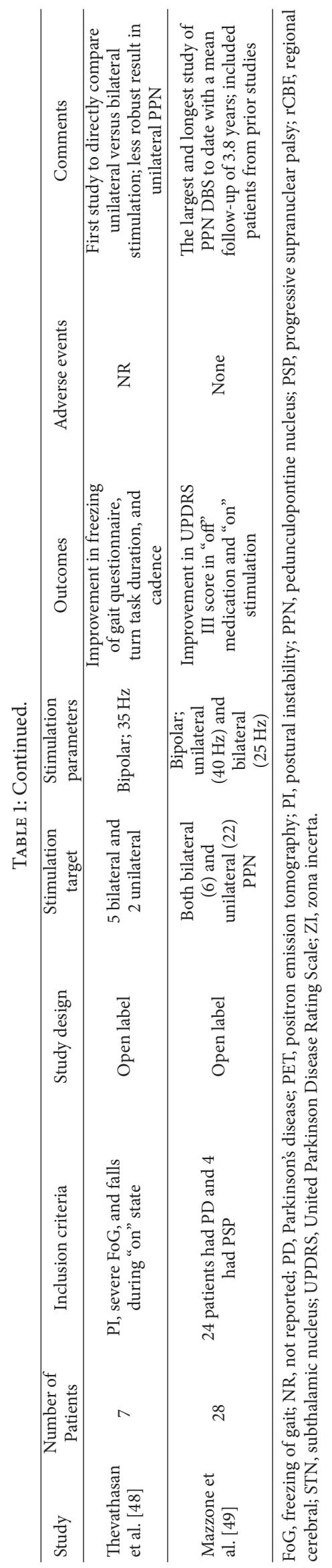


et al. were the first to investigate the role of unilateral PPNDBS in a double-blinded study of 6 patients with PD [44]. At study end period (1 year), UPDRS item 13 (falling) showed $75 \%$ improvement, with no statistically significant changes in other motor domains. Furthermore, bilateral stimulation proved more effective than unilateral stimulation [48].

The largest study with the longest follow-up of PPN-DBS in PD was reported by Mazzone et al. [49, 68, 69]. A total of 24 patients with PD and 4 with progressive supranuclear palsy (PSP) [49] were followed for a mean follow-up of 3.8 years. At study end period, they demonstrated an improvement in UPDRS III scores and in axial symptoms (UPDRS items 27-30) (off levodopa therapy); however, no difference was detected between the "on" medication and "off" stimulation state and the "off" medication and "on" stimulation state.

Connectivity to and from the MLR/PPN appears critical for normal gait function and is likely a factor in FoG as well. Structural deficits in connectivity are evident between the basal ganglia and PPN, in addition to other tracts in patients with FoG [70, 71]. Functional connectivity studies suggest that FoG patients may have significantly stronger connectivity between the PPN and supplementary motor area (SMA) [70], possibly reflecting maladaptive compensatory mechanisms. The integrity of these tracts has not been studied in patients who have undergone PPN-DBS. The variability of this deficit in structural and functional connectivity to and from the PPN may at least partially explain the variable results within the literature. In addition, the PPN tends to be spatially diffuse in humans and microelectrode recording is not helpful intraoperatively, thus making precise lead placement difficult and potentially contributing to further variability from study to study.

The experience and results with PPN-DBS are in their infancy. More precise targeting strategies with improved technology (i.e., improved imaging and programming) are required. It remains to be seen whether PPN-DBS should be an adjunct target to STN- or GPi-DBS for better overall motor control.

2.2.2. Combined Pedunculopontine Nucleus and Caudal Zona Incerta Stimulation. Khan et al. investigated the effects of bilateral PPN-DBS and caudal CZI-DBS in a blinded study of 7 patients with PD [47]. The authors demonstrated an $18.8 \%$ improvement in UPDRS III score and a $26.3 \%$ improvement in axial symptoms (items 27-30 on UPDRS III) of levodopa therapy. However, the same subscore was only significantly reduced in the "on" medication state when the PPN and cZI were stimulated in concert. This study suggested that, with these stimulation parameters, PPN stimulation alone was insufficient in improving "on" medication and resistant axial symptoms and that costimulation of cZI could provide an additive, beneficial role.

2.2.3. Substantia Nigra Pars Reticulata. The SN is a dense, laterally oriented collection of dopaminergic and GABAergic neurons located within the ventral midbrain, just dorsal to the corticospinal and corticobulbar tracts, ventral to the red nuclei, and lateral to the ventral tegmental area [72]. Its 2 components, the SNr and SNc [63], have traditionally been considered the major output and input nuclei, respectively, of the basal ganglia. While there is fair overlap, the $\mathrm{SNr}$ lies ventrally and laterally to the SNc in the midbrain [73]. In the classically held framework of basal ganglia circuitry, facilitation of movement was felt to be achieved through activation of a direct pathway from striatum to output nuclei (SNr and GPi), while inhibition of movement occurred through excitation of an indirect pathway (through globus pallidus externus and STN) [72]; however, recent advances in modeling of striatonigral-thalamocortical pathways have made it clear that while the classical model of basal ganglia circuitry provides a solid foundation for the understanding of its complex interconnections, it hardly captures its complete intricacies [72].

The $\mathrm{SNr}$ is another key player in the MLR, via its significant efferent GABAergic input to the PPN [74]. Efferents from the lateral $\mathrm{SNr}$ to the PPN are felt to modulate postural tone, while its medial efferents projecting to the cuneiform nucleus of the MLR influence locomotion [74]. It may not then be surprising that axial motor symptomatology, including gait impairment and PI, in patients with PD has shown favorable response to $\mathrm{SNr}$ stimulation in the literature [55-57, 75] (Table 2). Significant improvements in UPDRS III axial motor subscores and braking capacity, but not in distal motor symptoms (segmental akinesia, limb rigidity, and tremor), have been observed previously with SNr-DBS [55]. In contrast, one of the more recent double-blind, cross-over, randomized controlled trials with combined STN and SNr stimulation did show significant improvement in FoG, but not in other axial symptoms when compared to STN-DBS alone [56]. With SNr-DBS, one should be cautious about the possibility of worsening akinesia, as increased immobility and recurrent falls were reported in 1 patient in the same study during the last week of follow-up under combined STN and SNr stimulation [56].

While some benefit from SN stimulation has been reported, significant and variable impacts on mood and behavior can occur, likely owing to its limbic projections $[76,77]$. Reports of acute depression [78, 79], hypomania [77], and mania $[76,80]$ secondary to high frequency $\mathrm{SN}$ stimulation are evident in the literature. While it is difficult to rule out STN participation in the provocation of mood symptoms, it is clear that stimulation of more ventrally placed leads within the $\mathrm{SN}$ and likely the $\mathrm{SNr}$ can preferentially elicit these symptoms.

2.2.4. Motor Cortex. Extradural motor cortex stimulation (EMCS) has been studied as another treatment modality in $\mathrm{PD}$, particularly for those patients with advanced PD who are poor surgical candidates [81-86]. The primary motor cortex is a key component of corticobasal ganglia loops and thus forms a potential therapeutic target in PD [87]. Tremor and rigidity in PD can be suppressed by EMCS [58, 88], and benefit has been seen in advanced PD $[82,83]$. Since initial reports, numerous studies have investigated the role of EMCS for the treatment of advanced PD, with variable results [58$60,84,86,89-91$ ] (Table 2). 


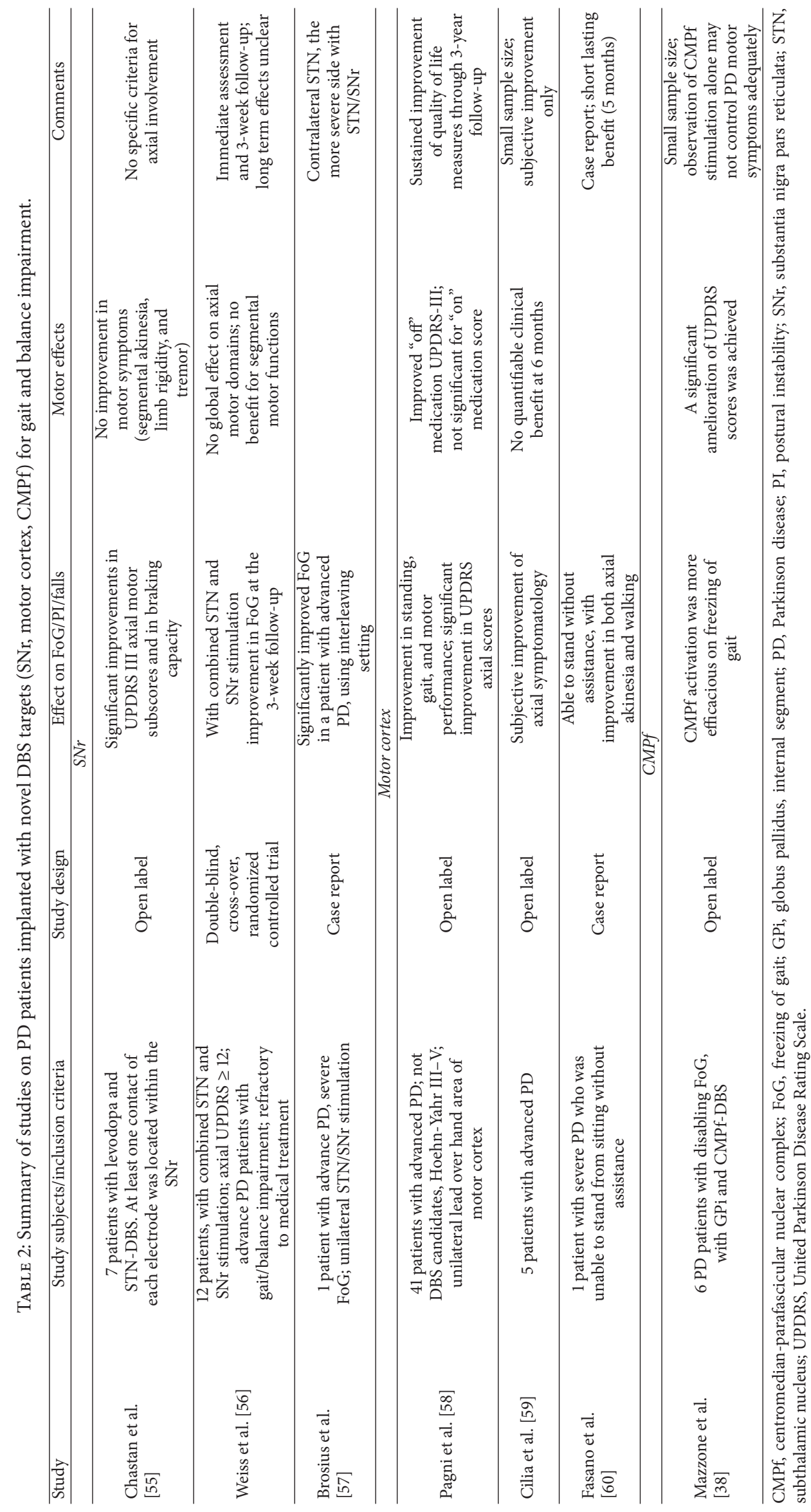


The largest study of EMCS in 41 patients with advanced PD (not eligible for DBS) showed improvement in standing, gait, and motor performance [58], though these results were not supported by other studies $[59,60,91]$. Additional studies have shown that EMCS improved quality of life parameters and modestly reduced levodopa dose but did not improve UPDRS III scores or axial symptoms [90, 92].

2.2.5. Centromedian and Parafascicular Nuclei. A single study demonstrated that CMPf stimulation alone led to significantly reduced FoG, where GPi stimulation alone did not [38]; however, this study had a sample size of only 6 patients. The authors further observed that CMPf stimulation alone may not control PD motor symptoms adequately. This observation raised the possibility of multiple-target stimulation strategy to optimize axial symptoms and overall motor control in PD.

2.3. Refractory Axial Symptoms-Speech and Swallowing. To date, no convincing evidence has demonstrated improvements in speech or swallowing in PD with STN- or GPiDBS. Speech and swallowing can worsen with DBS surgery or programming. Research on the impact of cZI-DBS on associated motor symptomatology in PD has also taken place. Particular focus in the literature has been given to the effects of cZI-DBS on speech and its related domains. Stimulation of cZI was shown to have a deleterious effect on voice intensity when compared to STN-DBS [93], while articulatory precision of speech also worsens in patients receiving cZI-DBS [94]. Significant impairment in verbal fluency is also observed in the immediate postoperative period; however, this deficit does not maintain significance in the long term [95]. Speech intelligibility has been demonstrated to be significantly reduced in cZI-DBS patients speaking from a read-speech passage [96]; however, this effect was not reproduced when evaluated from spontaneous speech at 1 year postoperatively, suggesting that the impact of cZI-DBS on speech intelligibility may have initially been overstated [97]. While STN-DBS has beneficial effects on pitch variability and range, cZI-DBS displayed no such benefit in a small study of 16 patients with 1-year followup [98]. The effect of cZI-DBS on swallowing dysfunction has also been evaluated in 2 longitudinal, prospective studies of 8 and 9 patients $[99,100]$. Both studies demonstrated that cZI-DBS did not have a clinically significant impact on either swallowing function or self-reported swallowingspecific quality of life at 1 year postoperatively. Further studies should help clarify the effect of cZI-DBS on both speech and swallowing dysfunction. In 1 study of EMCS in advanced PD, Pagni et al. demonstrated improved speech and swallowing in patients who are not DBS candidates [58].

Speech and swallowing symptoms following DBS have yet to be defined within the current literature. Methodology in assessing the symptoms varies from study to study. Severity of dysarthria/dysphagia preoperatively, duration and severity of disease, and positioning of the electrode(s) are all critical contributing factors in speech outcomes. Large-scale studies and systemic analyses are required.

\section{Nonmotor Symptoms of PD}

NMS are debilitating in PD. Robust evidence is lacking for STN- and GPi-DBS in treating NMS. A number of reports have demonstrated that PPN-DBS is capable of modulating the NMS of PD, including cognition, sleep, and attention [101-103]. The cognitive benefit of PPN-DBS has been reported in a small number of uncontrolled studies, with bilateral PPN stimulation reducing reaction time when assessing executive function and working memory and improving delayed recall and verbal fluency [101, 102]. It has been postulated that the cognitive improvement in these domains might be mediated via activation of ascending cholinergic neurons to the thalamic CMPf, subsequently leading to widespread activation via intralaminar thalamic nuclei. Indeed, functional imaging via positron emission tomography has shown an increase in fluorodeoxyglucose uptake in prefrontal areas, suggesting a modulation of thalamic metabolism after PPN-DBS [104]. Romigi was the first to identify the role of PPN-DBS in sleep, demonstrating that bilateral PPN stimulation resulted in increased rapid eye movement (REM) sleep in patients with PD [105]. Similarly, Lim et al. showed that unilateral PPN-DBS in 3 PD patients and 2 PSP patients resulted in increased nocturnal REM sleep [106]. In a subsequent study by the same group, the authors noted that bilateral, low-frequency stimulation of the PPN resulted in improved attention in 2 patients with PD [107]. No other studies to date have investigated the role of PPN-DBS in attention.

DBS targets involved in memory circuits have garnered interest in recent years. To date, only 1 human study of DBS with bilateral STN and nucleus basalis of Meynert (NBM) stimulation in PD dementia (PDD) has evaluated the potential for cognitive and/or memory improvement [108]. In this study, STN-DBS alone yielded significant improvements in motor functioning, but not in memory or cognition. The addition of NBM stimulation to STN stimulation produced significant improvements in memory and cognitive functioning, manifested as improved performance on the Rey Auditory Verbal Learning Task, Trail-Making Test A, and the Clock Drawing Test.

\section{Discussion}

A multitude of new developments have been made in the area of alternative DBS targets in PD treatment over the last two decades. Research has focused on novel DBS targets, with the aim of relieving motor symptoms and NMS that are usually refractory to dopaminergic agents and traditional STN-, GPi-, and VIM-DBS.

Stimulation of the cZI has shown promise in alleviating severe parkinsonian tremor, amongst other types, and its costimulation with PPN could provide an additive benefit on axial symptoms and PI. cZI stimulation is relatively new in its conception and additional studies are required to further evaluate its possible deleterious effects on speech, particularly voice intensity and articulatory precision.

Studies investigating axial motor symptomatology and PI with PPN stimulation have yielded mixed results. From 
a technical aspect, considerable variability exists amongst stimulation parameters in PPN-DBS studies (Table 1) and may account for the variable degrees of success in relieving axial motor symptoms. Additionally, the PPN tends to be spatially diffuse in humans and electrophysiological recording intraoperatively is not as helpful [109] as that of the STN, GPi, or VIM. The connectivity deficit of the PPN should also be taken into account with invasive procedures like DBS. White matter tract integrity may prove fruitful with respect to patient selection. With regard to study design, a PD population with clear dopamine-resistant gait and balance deficits should be chosen. Moreover, whether or not study subjects have concurrent STN- or GPi-DBS should be considered and studied systemically to verify the therapeutic benefit of PPN stimulation. As indicated in Table 1, few studies have been randomized and double-blinded. High quality randomized studies with standardized outcomes are needed.

The SNr represents an area of great importance in the complex hierarchy of basal ganglia circuitry and studies evaluating its potential as a DBS target have yielded mixed results. While some studies of SNr-DBS have shown improvement in axial motor symptoms, the incidence of acute mania, hypomania, and depression suggests that its utility as a target in alleviating PD symptoms may be limited by these adverse changes.

EMCS and CMPf-DBS provide some benefit in PD symptomatology. However, evidence is not conclusive for either target to be superior to STN or GPi in motor control.

NMS symptoms are disabling in PD patients. Although there is some evidence that PPN-DBS improves NMS, data are as of yet too limited to consider PPN-DBS as a therapeutic option for this domain of symptomatology. PPN-DBS may prove to be a safer target in the cognitive domain, particularly when considering the possible impact of STN- and GPi-DBS on cognition.

\section{Conclusions}

The future of DBS in PD appears promising. The field has advanced significantly with a number of new targets to address the refractory symptoms of PD. Amongst the studies investigating these novel targets, the large majority are open-label and are not powerful enough to determine true therapeutic benefit. Future, large-scale randomized studies focusing on identifying ideal candidates, optimal targets, and stimulation parameters would certainly be of utility in triggering the DBS community to perform more robust comparisons across studies.

\section{Abbreviations}

cZI: Caudal zona incerta

CMPf: Centromedian-parafascicular nuclear complex

DBS: Deep brain stimulation

EMCS: Extradural motor cortex stimulation

FoG: Freezing of gait

GPi: Globus pallidus pars interna

MLR: Mesencephalic locomotor region
MPTP: 1-Methyl-4-phenyl-1,2,3,6-tetrahydropyridine

NBM: Nucleus basalis of Meynert

NMS: Nonmotor symptoms

PD: $\quad$ Parkinson's disease

PI: $\quad$ Postural instability

PPN: Pedunculopontine nucleus

PSA: Posterior subthalamic area

PSP: $\quad$ Progressive supranuclear palsy

REM: Rapid eye movement

SMA: Supplementary motor area

SN: $\quad$ Substantia nigra

SNc: Substantia nigra pars compacta

SNr: Substantia nigra pars reticulata

STN: Subthalamic nucleus

UPDRS: Unified Parkinson's Disease Rating Scale

VIM: Ventral intermediate nucleus

VOA: Ventralis oralis anterior

ZI: Zona incerta.

\section{Conflicts of Interest}

The authors declare no conflicts of interest.

\section{Authors' Contributions}

Dustin Anderson and Grayson Beecher contributed equally to this work.

\section{References}

[1] A. L. Benabid, P. Pollak, A. Louveau, S. Henry, and J. De Rougemont, "Combined (thalamotomy and stimulation) stereotactic surgery of the vim thalamic nucleus for bilateral parkinson disease," Stereotactic and Functional Neurosurgery, vol. 50, no. 1-6, pp. 344-346, 1987.

[2] A. Fasano, L. M. Romito, A. Daniele et al., "Motor and cognitive outcome in patients with Parkinson's disease 8 years after subthalamic implants," Brain, vol. 133, no. 9, pp. 2664-2676, 2010.

[3] M. G. Rizzone, A. Fasano, A. Daniele et al., "Long-term outcome of subthalamic nucleus DBS in Parkinson's disease: from the advanced phase towards the late stage of the disease?" Parkinsonism and Related Disorders, vol. 20, no. 4, pp. 376-381, 2014.

[4] A. Castrioto, A. M. Lozano, Y.-Y. Poon, A. E. Lang, M. Fallis, and E. Moro, "Ten-year outcome of subthalamic stimulation in Parkinson disease: A blinded evaluation," Archives of Neurology, vol. 68, no. 12, pp. 1550-1556, 2011.

[5] G. Cossu and M. Pau, "Subthalamic nucleus stimulation and gait in Parkinson's Disease: a not always fruitful relationship," Gait Posture, vol. 52, pp. 205-210, 2017.

[6] G. Guzzi, "Critical reappraisal of DBS targeting for movement disorders," Journal of Neurosurgical Sciences, vol. 60, no. 2, pp. 181-188, 2016.

[7] A. Collomb-Clerc and M.-L. Welter, "Effects of deep brain stimulation on balance and gait in patients with Parkinson's disease: a systematic neurophysiological review," Neurophysiologie Clinique, vol. 45, no. 4-5, pp. 371-388, 2015.

[8] M. S. Okun, "Subthalamic deep brain stimulation with a constant-current device in Parkinson's disease: an open-label 
randomised controlled trial," Lancet Neurol, vol. 11, no. 2, pp. 140-149, 2012.

[9] M. C. Rodriguez-Oroz, J. A. Obeso, A. E. Lang et al., "Bilateral deep brain stimulation in Parkinson's disease: A multicentre study with 4 years follow-up," Brain, vol. 128, no. 10, pp. 22402249, 2005.

[10] K. A. Follett, "Pallidal versus subthalamic deep-brain stimulation for Parkinson's disease," New England Journal of Medicine, vol. 362, no. 22, pp. 2077-2091.

[11] B. F. L. van Nuenen, R. A. J. Esselink, M. Munneke, J. D. Speelman, T. van Laar, and B. R. Bloem, "Postoperative gait deterioration after bilateral subthalamic nucleus stimulation in Parkinson's disease," Movement Disorders, vol. 23, no. 16, pp. 2404-2406, 2008.

[12] M. U. Ferraye, B. Debû, V. Fraix et al., "Effects of subthalamic nucleus stimulation and levodopa on freezing of gait in Parkinson disease," Neurology, vol. 70, no. 16, pp. 1431-1437, 2008.

[13] M. E. McNeely and G. M. Earhart, "Medication and subthalamic nucleus deep brain stimulation similarly improve balance and complex gait in Parkinson disease," Parkinsonism and Related Disorders, vol. 19, no. 1, pp. 86-91, 2013.

[14] P. Crenna, I. Carpinella, M. Rabuffetti et al., "Impact of subthalamic nucleus stimulation on the initiation of gait in Parkinson's disease," Experimental Brain Research, vol. 172, no. 4, pp. 519532, 2006.

[15] H. Stolze, S. Klebe, M. Poepping et al., "Effects of bilateral subthalamic nucleus stimulation on parkinsonian gait," Neurology, vol. 57, no. 1, pp. 144-146, 2001.

[16] F. Xu et al., "Deep brain stimulation of pallidal versus subthalamic for patients with Parkinson's disease: a meta-analysis of controlled clinical trials," Neuropsychiatr Dis Treat, vol. 12, pp. 1435-1444, 2016.

[17] S. Alomar, N. K. King, J. Tam, A. A. Bari, C. Hamani, and A. M. Lozano, "Speech and language adverse effects after thalamotomy and deep brain stimulation in patients with movement disorders: A meta-analysis," Movement Disorders, vol. 32, no. 1, pp. 53-63, 2017.

[18] D. Aldridge, D. Theodoros, A. Angwin, and A. P. Vogel, "Speech outcomes in parkinson's disease after subthalamic nucleus deep brain stimulation: a systematic review," Parkinsonism and Related Disorders, vol. 33, pp. 3-11, 2016.

[19] G. Deuschl, J. Herzog, G. Kleiner-Fisman et al., "Deep brain stimulation: postoperative issues," Movement Disorders, vol. 21, 14, pp. S219-S237, 2006.

[20] M. S. Troche, A. E. Brandimore, K. D. Foote, and M. S. Okun, "Swallowing and deep brain stimulation in parkinson's disease: a systematic review," Parkinsonism and Related Disorders, vol. 19, no. 9, pp. 783-788, 2013.

[21] L. W. J. Baijens and R. Speyer, "Effects of therapy for dysphagia in parkinson's disease: Systematic review," Dysphagia, vol. 24, no. 1, pp. 91-102, 2009.

[22] M. A. Hely, W. G. J. Reid, M. A. Adena, G. M. Halliday, and J. G. L. Morris, "The Sydney multicenter study of parkinson's disease: the inevitability of dementia at 20 years," Movement Disorders, vol. 23, no. 6, pp. 837-844, 2008.

[23] P. Blomstedt, A. Fytagoridis, M. Åström, J. Linder, L. Forsgren, and M. I. Hariz, "Unilateral caudal zona incerta deep brain stimulation for parkinsonian tremor," Parkinsonism and Related Disorders, vol. 18, no. 10, pp. 1062-1066, 2012.

[24] P. Blomstedt, A. Fytagoridis, and S. Tisch, "Deep brain stimulation of the posterior subthalamic area in the treatment of tremor," Acta Neurochirurgica, vol. 151, no. 1, pp. 31-36, 2009.
[25] P. Blomstedt, U. Sandvik, A. Fytagoridis, and S. Tisch, "The posterior subthalamic area in the treatment of movement disorders: past, present, and future," Neurosurgery, vol. 64, no. 6, pp. 1029-1038, 2009.

[26] J. Mitrofanis, "Some certainty for the "zone of uncertainty"? exploring the function of the zona incerta," Neuroscience, vol. 130, no. 1, pp. 1-15, 2005.

[27] P. Plaha, S. Khan, and S. S. Gill, "Bilateral stimulation of the caudal zona incerta nucleus for tremor control," Journal of Neurology, Neurosurgery and Psychiatry, vol. 79, no. 5, pp. 504513, 2008.

[28] A. Chopra, B. T. Klassen, and M. Stead, "Current clinical application of deep-brain stimulation for essential tremor," Neuropsychiatric Disease and Treatment, vol. 9, pp. 1859-1865, 2013.

[29] N. K. Patel, P. Heywood, K. O’Sullivan, R. McCarter, S. Love, and S. S. Gill, "Unilateral subthalamotomy in the treatment of parkinson's disease," Brain, vol. 126, no. 5, pp. 1136-1145, 2003.

[30] P. Plaha, Y. Ben-Shlomo, N. K. Patel, and S. S. Gill, "Stimulation of the caudal zona incerta is superior to stimulation of the subthalamic nucleus in improving contralateral parkinsonism," Brain, vol. 129, no. 7, pp. 1732-1747, 2006.

[31] A. M. Burrows, P. D. Ravin, P. Novak et al., "Limbic and motor function comparison of deep brain stimulation of the zona incerta and subthalamic nucleus," Operative Neurosurgery, vol. 70, 1, pp. 125-130, 2012.

[32] E. E. Benarroch, "The midline and intralaminar thalamic nuclei: anatomic and functional specificity and implications in neurologic disease," Neurology, vol. 71, no. 12, pp. 948-949, 2008.

[33] K. D. Foote, P. Seignourel, H. H. Fernandez et al., "Dual electrode thalamic deep brain stimulation for the treatment of posttraumatic and multiple sclerosis tremor," Neurosurgery, vol. 58, no. 2, pp. S-280-S-285, 2006.

[34] G. Broggi, C. Giorgi, and D. Servello, "Stereotactic neurosurgery in the treatment of tremor," Acta Neurochir Suppl (Wien), vol. 39, pp. 73-76, 1987.

[35] J. K. Krauss et al., "Deep brain stimulation of the centre medianparafascicular complex in patients with movement disorders," Journal of Neurology, Neurosurgery \& Psychiatry, vol. 72, no. 4, pp. 546-548, 2002.

[36] A. Stefani, A. Peppe, and M. Pierantozzi, "Multi-target strategy for Parkinsonian patients: the role of deep brain stimulation in the centromedian-parafascicularis complex," Brain Research Bulletin, vol. 78, no. 2-3, pp. 113-118, 2009.

[37] A. Peppe, A. Gasbarra, A. Stefani et al., "Deep brain stimulation of $\mathrm{CM} / \mathrm{PF}$ of thalamus could be the new elective target for tremor in advanced parkinson's disease?" Parkinsonism and Related Disorders, vol. 14, no. 6, pp. 501-504, 2008.

[38] P. Mazzone, F. Stocchi, S. Galati et al., "Bilateral implantation of centromedian-parafascicularis complex and GPi: a new combination of unconventional targets for deep brain stimulation in severe parkinson disease," Neuromodulation, vol. 9, no. 3, pp. 221-228, 2006.

[39] P. Mazzone, A. Lozano, P. Stanzione et al., "Implantation of human pedunculopontine nucleus: a safe and clinically relevant target in parkinson's disease," NeuroReport, vol. 16, no. 17, pp. 1877-1881, 2005.

[40] P. Plaha and S. S. Gill, "Bilateral deep brain stimulation of the pedunculopontine nucleus for parkinson's disease," NeuroReport, vol. 16, no. 17, pp. 1883-1887, 2005. 
[41] A. Stefani, A. M. Lozano, A. Peppe et al., "Bilateral deep brain stimulation of the pedunculopontine and subthalamic nuclei in severe parkinson's disease," Brain, vol. 130, part 6, pp. 1596-1607, 2007.

[42] A. P. Strafella, A. M. Lozano, B. Ballanger, Y.-Y. Poon, A. E. Lang, and E. Moro, "rCBF changes associated with PPN stimulation in a patient with parkinson's disease: A PET study," Movement Disorders, vol. 23, no. 7, pp. 1051-1054, 2008.

[43] M. U. Ferraye, B. Debû, and V. Fraix, "Effects of pedunculopontine nucleus area stimulation on gait disorders in parkinson's disease," Brain, vol. 133, part 1, pp. 205-214, 2010.

[44] E. Moro, C. Hamani, Y.-Y. Poon et al., "Unilateral pedunculopontine stimulation improves falls in parkinson's disease," Brain, vol. 133, no. 1, pp. 215-224, 2010.

[45] W. Thevathasan, P. A. Silburn, and H. Brooker, "The impact of low-frequency stimulation of the pedunculopontine nucleus region on reaction time in parkinsonism," Journal of Neurology, Neurosurgery and Psychiatry, vol. 81, no. 10, pp. 1099-1104, 2010.

[46] W. Thevathasan, T. J. Coyne, J. A. Hyam et al., "Pedunculopontine nucleus stimulation improves gait freezing in parkinson disease," Neurosurgery, vol. 69, no. 6, pp. 1248-1253, 2011.

[47] S. Khan, L. Mooney, P. Plaha et al., "Outcomes from stimulation of the caudal zona incerta and pedunculopontine nucleus in patients with parkinson's disease," British Journal of Neurosurgery, vol. 25, no. 2, pp. 273-280, 2011.

[48] W. Thevathasan, A. Pogosyan, J. A. Hyam et al., "Alpha oscillations in the pedunculopontine nucleus correlate with gait performance in parkinsonism," Brain, vol. 135, no. 1, pp. 148160, 2012.

[49] P. Mazzone, S. Sposato, A. Insola, and E. Scarnati, “The clinical effects of deep brain stimulation of the pedunculopontine tegmental nucleus in movement disorders may not be related to the anatomical target, leads location, and setup of electrical stimulation," Neurosurgery, vol. 73, no. 5, pp. 894-906, 2013.

[50] Y. Okuma, "Freezing of gait and falls in parkinson's disease," Journal of Parkinson's Disease, vol. 4, no. 2, pp. 255-260, 2014.

[51] S. Perez-Lloret, L. Negre-Pages, P. Damier et al., "Prevalence, determinants, and effect on quality of life of freezing of gait in Parkinson disease," The JAMA Neurology, vol. 71, no. 7, pp. 884890, 2014.

[52] C. A. Z. Coste, "Detection of freezing of gait in Parkinson disease: preliminary results," Sensors (Basel, Switzerland), vol. 14, no. 4, pp. 6819-6827, 2014.

[53] J. D. Schaafsma, Y. Balash, T. Gurevich, A. L. Bartels, J. M. Hausdorff, and N. Giladi, "Characterization of freezing of gait subtypes and the response of each to levodopa in Parkinson's disease," European Journal of Neurology, vol. 10, no. 4, pp. 391398, 2003.

[54] R. Chee, A. Murphy, M. Danoudis, N. Georgiou-Karistianis, and R. Iansek, "Gait freezing in parkinson's disease and the stride length sequence effect interaction," Brain, vol. 132, no. 8, pp. 2151-2160, 2009.

[55] N. Chastan, G. W. M. Westby, J. Yelnik et al., "Effects of nigral stimulation on locomotion and postural stability in patients with parkinson's disease," Brain, vol. 132, no. 1, pp. 172-184, 2009.

[56] D. Weiss, M. Walach, C. Meisner et al., "Nigral stimulation for resistant axial motor impairment in parkinson's disease? a randomized controlled trial," Brain, vol. 136, no. 7, pp. 20982108, 2013.

[57] S. N. Brosius, C. L. Gonzalez, J. Shuresh, and H. C. Walker, "Reversible improvement in severe freezing of gait from parkinson's disease with unilateral interleaved subthalamic brain stimulation," Parkinsonism and Related Disorders, vol. 21, no. 12, pp. 1469-1470, 2015.

[58] C. A. Pagni et al., "Results by motor cortex stimulation in treatment of focal dystonia, parkinson's disease and post-ictal spasticity. The experience of the italian study group of the italian neurosurgical society," Acta Neurochir Suppl, vol. 101, pp. 13-21, 2008.

[59] R. Cilia, A. Landi, F. Vergani, E. Sganzerla, G. Pezzoli, and A. Antonini, "Extradural motor cortex stimulation in parkinson's sisease," Movement Disorders, vol. 22, no. 1, pp. 111-114, 2007.

[60] A. Fasano, C. Piano, C. De Simone et al., "High frequency extradural motor cortex stimulation transiently improves axial symptoms in a patient with parkinson's disease," Movement Disorders, vol. 23, no. 13, pp. 1916-1919, 2008.

[61] M. S. Lee, J. O. Rinne, and C. D. Marsden, "The pedunculopontine nucleus: its role in the genesis of movement disorders.", Yonsei Medical Journal, vol. 41, no. 2, pp. 167-184, 2000.

[62] T. L. Tattersall, P. G. Stratton, T. J. Coyne et al., "Imagined gait modulates neuronal network dynamics in the human pedunculopontine nucleus," Nature Neuroscience, vol. 17, no. 3, pp. 449-454, 2014.

[63] I. Guella et al., "Alpha-synuclein genetic variability: a biomarker for dementia in parkinson disease," Ann Neurol, vol. 79, no. 6, pp. 991-999, 2016.

[64] J. O. Rinne, S. Y. Ma, M. S. Lee, Y. Collan, and M. Röyttä, "Loss of cholinergic neurons in the pedunculopontine nucleus in parkinson's disease is related to disability of the patients," Parkinsonism and Related Disorders, vol. 14, no. 7, pp. 553-557, 2008.

[65] N. Jenkinson, D. Nandi, R. C. Miall, J. F. Stein, and T. Z. Aziz, "Pedunculopontine nucleus stimulation improves akinesia in a Parkinsonian monkey," NeuroReport, vol. 15, no. 17, pp. 26212624, 2004.

[66] W. Thevathasan, M. H. Cole, C. L. Graepel et al., "A spatiotemporal analysis of gait freezing and the impact of pedunculopontine nucleus stimulation," Brain, vol. 135, no. 5, pp. 1446-1454, 2012.

[67] W. Thevathasan, A. Pogosyan, J. A. Hyam et al., "A block to preprepared movement in gait freezing, relieved by pedunculopontine nucleus stimulation," Brain, vol. 134, no. 7, pp. 2085-2095, 2011.

[68] P. Mazzone, S. Sposato, A. Insola, V. Dilazzaro, and E. Scarnati, "Stereotactic surgery of nucleus tegmenti pedunculopontini," British Journal of Neurosurgery, vol. 22, no. 1, pp. S33-S40, 2008.

[69] P. Mazzone, S. Sposato, A. Insola, and E. Scarnati, "The deep brain stimulation of the pedunculopontine tegmental nucleus: towards a new stereotactic neurosurgery," Journal of Neural Transmission, vol. 118, no. 10, pp. 1431-1451, 2011.

[70] B. W. Fling, R. G. Cohen, M. Mancini et al., "Functional reorganization of the locomotor network in parkinson patients with freezing of gait," PLoS ONE, vol. 9, no. 6, Article ID e100291, 2014.

[71] S. Vercruysse, I. Leunissen, G. Vervoort, W. Vandenberghe, S. Swinnen, and A. Nieuwboer, "Microstructural changes in white matter associated with freezing of gait in parkinson's disease," Movement Disorders, vol. 30, no. 4, pp. 567-576, 2015.

[72] L. Yetnikoff, H. N. Lavezzi, R. A. Reichard, and D. S. Zahm, "An update on the connections of the ventral mesencephalic dopaminergic complex," Neuroscience, vol. 282, pp. 23-48, 2014.

[73] S. N. Haber, "The place of dopamine in the cortico-basal ganglia circuit," Neuroscience, vol. 282, pp. 248-257, 2014. 
[74] K. Takakusaki, R. Chiba, T. Nozu, and T. Okumura, "Brainstem control of locomotion and muscle tone with special reference to the role of the mesopontine tegmentum and medullary reticulospinal systems," Journal of Neural Transmission, vol. 123, no. 7, pp. 695-729, 2016.

[75] D. Weiss, R. Klotz, R. B. Govindan et al., "Subthalamic stimulation modulates cortical motor network activity and synchronization in parkinson's disease," Brain, vol. 138, no. 3, pp. 679-693, 2015.

[76] M. Ulla, S. Thobois, J.-J. Lemaire et al., "Manic behaviour induced by deep-brain stimulation in parkinson's disease: evidence of substantia nigra implication?" Journal of Neurology, Neurosurgery and Psychiatry, vol. 77, no. 12, pp. 1363-1366, 2006.

[77] M. Ulla, S. Thobois, P.-M. Llorca et al., "Contact-dependent reproducible hypomania induced by deep brain stimulation in Parkinson's disease: clinical, anatomical, and functional imaging study," Journal of Neurology, Neurosurgery and Psychiatry, vol. 82, no. 6, pp. 607-614, 2011.

[78] B.-P. Bejjani, P. Damier, I. Arnulf et al., "Transient acute depression induced by high-frequency deep-brain stimulation," The New England Journal of Medicine, vol. 340, no. 19, pp. 1476$1480,1999$.

[79] P. Blomstedt, M. I. Hariz, A. Lees et al., "Acute severe depression induced by intraoperative stimulation of the substantia nigra: a case report," Parkinsonism \& Related Disorders, vol. 14, no. 3, pp. 253-256, 2008.

[80] J. Kulisevsky, M. L. Berthier, A. Gironell, B. Pascual-Sedano, J. Molet, and P. Parés, "Mania following deep brain stimulation for parkinson's disease," Neurology, vol. 59, no. 9, pp. 1421-1424, 2002.

[81] E. Benvenuti, F. Cecchi, A. Colombini, and G. Gori, "Extradural motor cortex stimulation as a method to treat advanced parkinson's disease: New perspective in geriatric medicine," Aging Clinical and Experimental Research, vol. 18, no. 4, pp. 347-348, 2006.

[82] S. Canavero and R. Paolotti, "Extradural motor cortex stimulation for advanced Parkinson's disease: case report," Movement Disorders, vol. 15, no. 1, pp. 169-171, 2000.

[83] S. Canavero, R. Paolotti, V. Bonicalzi et al., "Extradural motor cortex stimulation for advanced parkinson disease: report of two cases," Journal of Neurosurgery, vol. 97, no. 5, pp. 1208-1211, 2002.

[84] B. Cioni, "Motor cortex stimulation for parkinson's disease," Acta Neurochir Suppl, vol. 97, no. 2, pp. 233-238, 2007.

[85] C. A. Pagni et al., "Extradural motor cortex stimulation (EMCS) for parkinson's disease. history and first results by the study group of the Italian neurosurgical society," Acta Neurochir Suppl, vol. 93, pp. 113-119, 2005.

[86] C. A. Pagni, S. Zeme, F. Zenga, and R. M. Villani, "Further experience with extradural motor cortex stimulation for treatment of advanced parkinson's disease: report of 3 new cases," Journal of Neurosurgical Sciences, vol. 47, no. 4, pp. 189-193, 2003.

[87] R. L. Albin, A. B. Young, and J. B. Penney, "The functional anatomy of disorders of the basal ganglia," Trends in Neurosciences, vol. 18, no. 2, pp. 63-64, 1995.

[88] C. N. Woolsey, T. C. Erickson, and W. E. Gilson, "Localization in somatic sensory and motor areas of human cerebral cortex as determined by direct recording of evoked potentials and electrical stimulation.," Journal of Neurosurgery, vol. 51, no. 4, pp. 476-506, 1979.

[89] J. E. Arle, D. Apetauerova, J. Zani et al., "Motor cortex stimulation in patients with Parkinson disease: 12-Month follow-up in
4 patients," Journal of Neurosurgery, vol. 109, no. 1, pp. 133-139, 2008 .

[90] J. C. Gutiérrez, F. J. Seijo, M. A. Á. Vega, F. F. González, B. L. Aragoneses, and M. Blázquez, "Therapeutic extradural cortical stimulation for parkinson's disease: report of six cases and review of the literature," Clinical Neurology and Neurosurgery, vol. 111, no. 8, pp. 703-707, 2009.

[91] E. Moro, J. M. Schwalb, P. Piboolnurak et al., "Unilateral subdural motor cortex stimulation improves essential tremor but not parkinson's disease," Brain, vol. 134, no. 7, pp. 2096-2105, 2011.

[92] A. R. Bentivoglio, A. Fasano, C. Piano et al., "Unilateral extradural motor cortex stimulation is safe and improves parkinson disease at 1 year," Neurosurgery, vol. 71, no. 4, pp. 815-825, 2012.

[93] S. Lundgren, T. Saeys, F. Karlsson et al., "Deep brain stimulation of caudal zona incerta and subthalamic nucleus in patients with parkinson's disease: effects on voice intensity," Parkinson's Disease, Article ID 658956, 2011.

[94] E. Eklund, J. Qvist, L. Sandström, F. Viklund, J. Van Doorn, and F. Karlsson, "Perceived articulatory precision in patients with parkinson's disease after deep brain stimulation of subthalamic nucleus and caudal zona incerta," Clinical Linguistics and Phonetics, vol. 29, no. 2, pp. 150-166, 2015.

[95] A. Fytagoridis, R. L. Sjöberg, M. Åström, A. Fredricks, L. Nyberg, and P. Blomstedt, "Effects of deep brain stimulation in the caudal zona incerta on verbal fluency," Stereotactic and Functional Neurosurgery, vol. 91, no. 1, pp. 24-29, 2013.

[96] L. Johansson, S. Möller, K. Olofsson et al., "Word-level intelligibility after caudal zona incerta stimulation for parkinson's disease," Acta Neurologica Scandinavica, vol. 130, no. 1, pp. 2733, 2014.

[97] L. Sandström, P. Hägglund, L. Johansson, P. Blomstedt, and F. Karlsson, "Speech intelligibility in parkinson's disease patients with zona incerta deep brain stimulation," Brain and Behavior, vol. 5, no. 10, 2015.

[98] F. Karlsson, K. Olofsson, P. Blomstedt, J. Linder, and J. van Doorn, "Pitch variability in patients with parkinson's disease: effects of deep brain stimulation of caudal zona incerta and subthalamic nucleus," Journal of Speech, Language, and Hearing Research, vol. 56, no. 1, pp. 150-158, 2013.

[99] S. Sundstedt et al., "Swallowing quality of life after zona incerta deep brain stimulation," Ann Otol Rhinol Laryngol, vol. 126, no. 2, pp. 110-116, 2017.

[100] S. Sundstedt, K. Olofsson, J. van Doorn, J. Linder, E. Nordh, and P. Blomstedt, "Swallowing function in parkinson's patients following Zona Incerta deep brain stimulation," Acta Neurologica Scandinavica, vol. 126, no. 5, pp. 350-356, 2012.

[101] S. Alessandro, R. Ceravolo, L. Brusa et al., "Non-motor functions in parkinsonian patients implanted in the pedunculopontine nucleus: Focus on sleep and cognitive domains," Journal of the Neurological Sciences, vol. 289, no. 1-2, pp. 44-48, 2010.

[102] A. Costa, G. A. Carlesimo, C. Caltagirone et al., "Effects of deep brain stimulation of the peduncolopontine area on working memory tasks in patients with parkinson's disease," Parkinsonism and Related Disorders, vol. 16, no. 1, pp. 64-67, 2010.

[103] H. Morita, C. J. Hass, E. Moro, A. Sudhyadhom, R. Kumar, and M. S. Okun, "Pedunculopontine nucleus stimulation: where are we now and what needs to be done to move the field forward?" Frontiers in Neurology, vol. 5, article no. 243, 2014. 
[104] R. Ceravolo, L. Brusa, S. Galati et al., "Low frequency stimulation of the nucleus tegmenti pedunculopontini increases cortical metabolism in Parkinsonian patients," European Journal of Neurology, vol. 18, no. 6, pp. 842-849, 2011.

[105] A. Romigi, "Pedunculopontine nucleus stimulation influences REM sleep in parkinson's disease," European Journal of Neurology, vol. 15, no. 7, pp. e64-e65, 2008.

[106] A. S. Lim, E. Moro, A. M. Lozano et al., "Selective enhancement of rapid eye movement sleep by deep brain stimulation of the human pons," Annals of Neurology, vol. 66, no. 1, pp. 110-114, 2009.

[107] I. Arnulf, M. Ferraye, V. Fraix et al., "Sleep induced by stimulation in the human pedunculopontine nucleus area," Annals of Neurology, vol. 67, no. 4, pp. 546-549, 2010.

[108] H. J. Freund et al., "Cognitive functions in a patient with Parkinson-dementia syndrome undergoing deep brain stimulation," Arch Neurol, vol. 66, no. 6, pp. 781-785, 2009.

[109] M. Weinberger, C. Hamani, W. D. Hutchison, E. Moro, A. M. Lozano, and J. O. Dostrovsky, "Pedunculopontine nucleus microelectrode recordings in movement disorder patients," Experimental Brain Research, vol. 188, no. 2, pp. 165-174, 2008. 


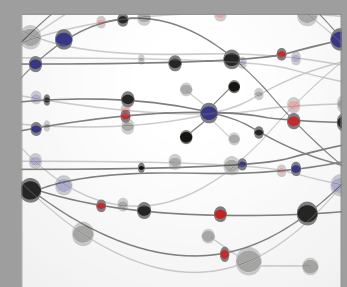

The Scientific World Journal
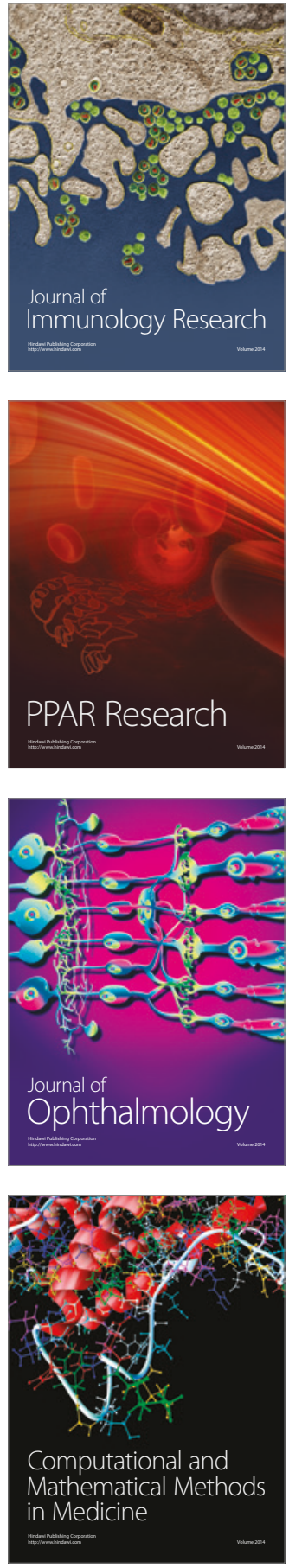

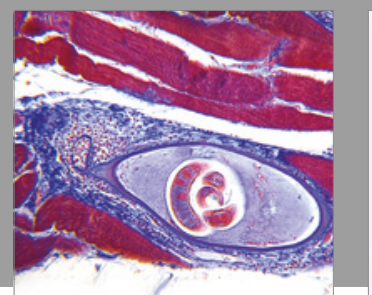

Gastroenterology Research and Practice
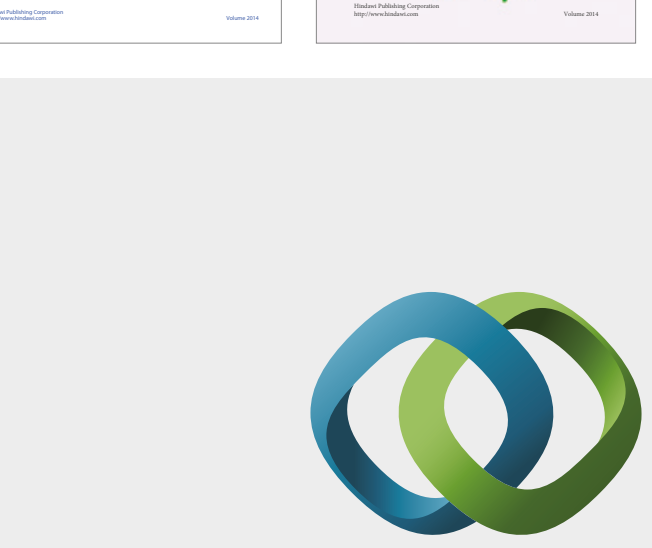

\section{Hindawi}

Submit your manuscripts at

https://www.hindawi.com
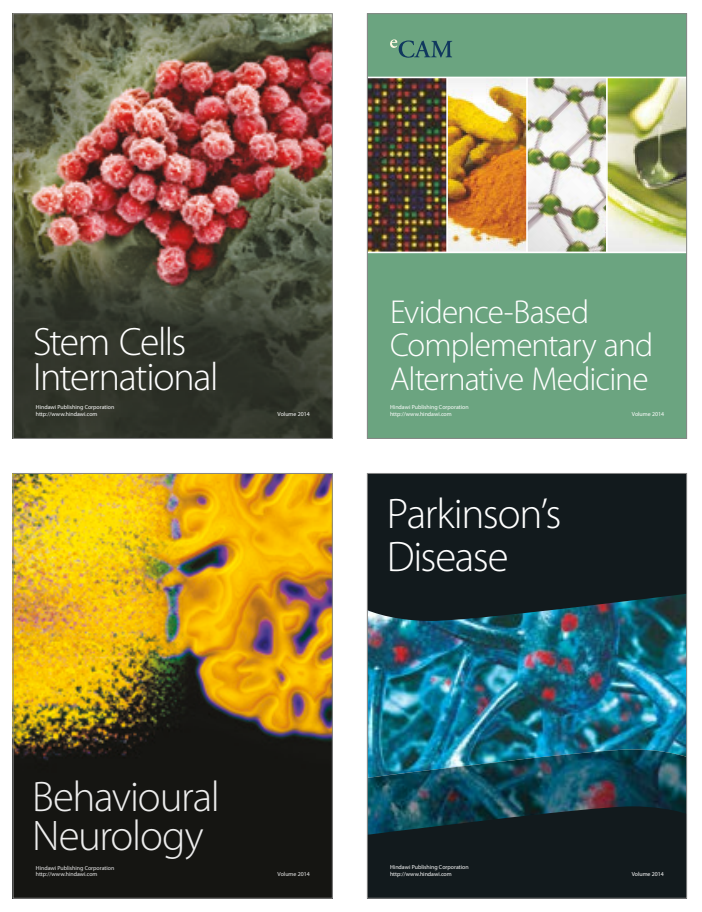
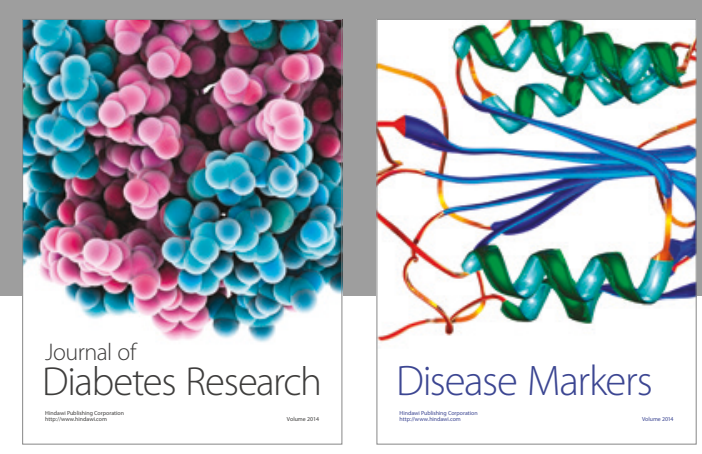

Disease Markers
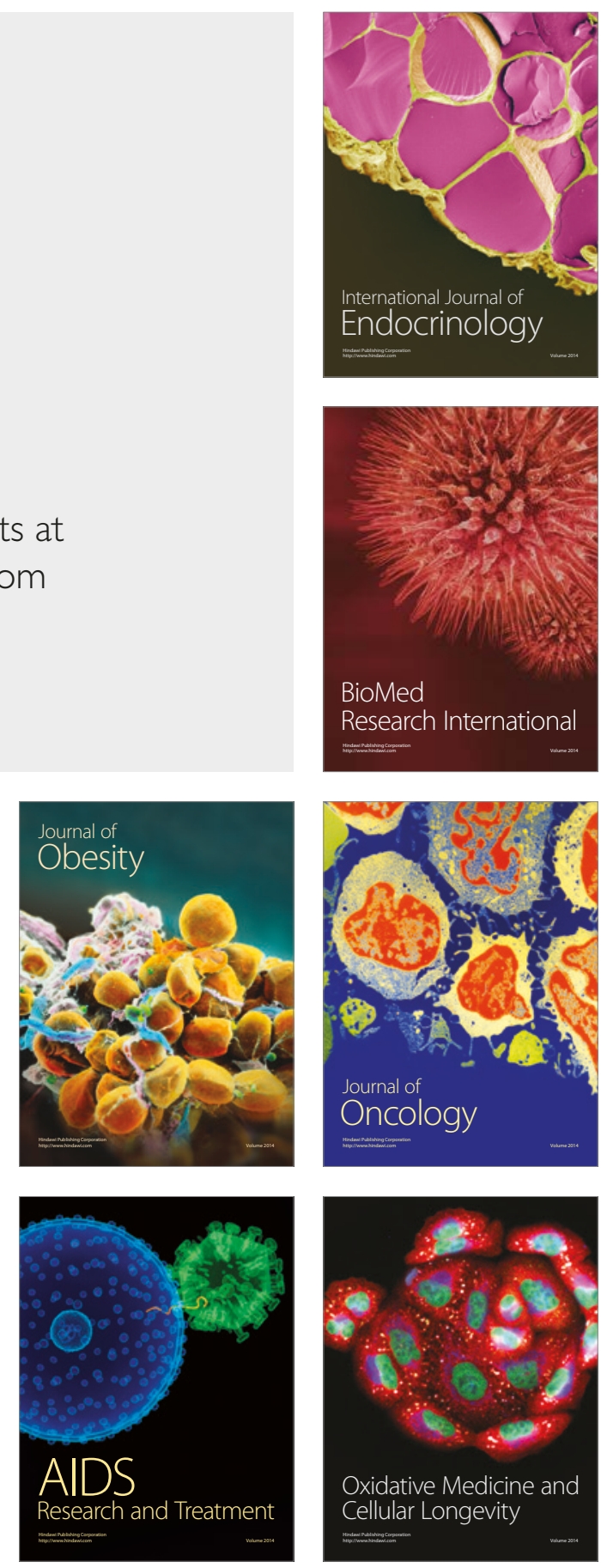